\title{
Investigation of Fluconazole Susceptibility to Candida Albicans by MALDI-TOF MS and Real-Time PCR for CDR1, CDR2, MDR1 and ERG11
}

Chanika Maenchantrarath

Thammasat University

Pradchama Khumdee

Thammasat University

Seksun Samosornsuk

Thammasat University

Narissara Mungkornkaew

Thammasat University

Worada Samosornsuk ( $\nabla$ sworada@hotmail.com )

Thammasat University

\section{Research Article}

Keywords: Candida albicans, MALDI-TOF MS, fluconazole susceptibility testing, CDR1, CDR2

Posted Date: July 8th, 2021

DOI: https://doi.org/10.21203/rs.3.rs-658761/v1

License: (9) This work is licensed under a Creative Commons Attribution 4.0 International License.

Read Full License 


\section{Abstract}

Background $C$. albicans is the most important yeast that caused the infection in humans; the trend of resistance to fluconazole (FLC) was also increased, while the FLC susceptibility by conventional method takes time causing the treatment failure. To investigate FLC susceptibility to $C$. albicans using MALDITOF MS and Real-time PCR for CDR1, CDR2, MDR1 and ERG11, overall, 32 C. albicans strains included 4 reference strains (3 FLC susceptible (S) and 1 FLC resistant (R), 1 spontaneous mutant strain (FLC susceptible-dose dependent, SDD) and 27 clinical strains obtained from 2 Thai University Hospitals were performed FLC susceptibility testing by Sensititre YeastOne and broth microdilution method, FLC resistant expression mechanism by Real-time PCR and the major peak determination by MALDI-TOF MS.

Results The change of $C D R 1$ and CDR2 mRNA expression were only significantly observed in SDD and R strains. Using MALDI-TOF MS, the change of mass spectral intensity at range $3376-3382 \mathrm{~m} / \mathrm{z}$ (major peak) was significantly related to FLC susceptibility as SDD (decreased at $4 \mu \mathrm{g} / \mathrm{ml}$ and increase at 8 $\mu \mathrm{g} / \mathrm{ml}$ ), S (all increased), and R (all slightly decreased or no change) after incubation for $6 \mathrm{~h}$. All 27 clinical strains showed FLC MIC susceptible (MIC range 0.25-2 $\mathrm{g} / \mathrm{ml}$ ), no change in CDR1 and CDR2 expression and $\mathrm{S}$ major peak type. The FLC resistance $C$. albicans with $C D R 1$ and $C D R 2$ expression may possibly effect the change of mass spectral intensity at range $3376-3382 \mathrm{~m} / \mathrm{z}$.

Conclusions The MALDI-TOF MS may be used to simultaneously classify and predict FLC resistant $C$. albicans strains associated with CDR1 and CDR2 expression. Further studies are essential to clarify the methodology and improve the reliability of this assay for routine diagnosis.

\section{Background}

Currently, the situation of fungal diseases especially candidemia in hospital is increasing the risk of morbidity and mortality [1]. Candida albicans is the most common reported species followed by $C$. glabrata, C. tropicalis, C. parapsilosis and C. krusei (Pichia kudriavzevii), respectively [2]. Fluconazole (FLC), a triazole antifungal is the most widely used for this infection. Fluconazole has a fungistatic effect by inhibiting cytochrome P450 enzyme lanosterol demethylase (14a-demethylase), encoded by ERG11, in the ergosterol biosynthesis pathway. Many $C$. albicans clinical isolates overexpress $E R G 11$, the gene encoding the target of the azoles. However, in many cases, the level of overexpression is minimal or else observed in combination with other resistance mutations, making it difficult to assess the direct impact of such overexpression on the resistant phenotype. In addition to ERG11 overexpression, two main classes of efflux proteins included ATP-binding cassette (ABC) transporters encoded by CDR1 and CDR2 and Major facilitator superfamily (MFS) encoded by MDR1 can mediate resistance to FLC by reducing the effective intracellular drug concentration [3, 4]. The azole resistance associated with ERG11, MDR or CDR overexpression has been reported in many cases $[5,6]$. For the time being, broth microdilution (BMD) method, a gold standard of antifungal susceptibility testing (AFST) of Candida spp. was standardized and determined MIC level of antifungal agents by Clinical and Laboratory Standards Institute (CLSI) and European Committee on Antibiotic Susceptibility Testing (EUCAST). Notwithstanding, this method is time- 
consuming and tedious steps. The matrix assisted laser desorption ionization-time of flight mass spectrometry (MALDI-TOF MS) is generally used for microbial identification by mass spectrum analysis compared with the databases [1]. Several studies were adapted the MALDI-TOF MS for testing susceptibility to bacteria and fungi AFST because of simple, high-throughput, waste reducing, rapid and low expense method [7-9]. The aim of this study was to investigate FLC susceptibility to $C$. albicans using MALDI-TOF MS and Real-time PCR for CDR1, CDR2, MDR1 and ERG11.

\section{Methods}

\section{Yeast Samples used in this study}

Clinical samples of $C$. albicans in this study were 15 strains collected from Microbiology Laboratory Unit, Department of Central Laboratory and Blood Bank, Faculty of Medicine, Vajira Hospital, Navamindradhiraj University, Bangkok, Thailand since April 2015 to December 2017, and 12 strains obtained from positive blood culture in Microbiology Laboratory, Thammasat University Hospital from January 2013 to April 2016. Four ATCC standard strains were used as the control strains including 2 susceptible strains (C. albicans MYA2876 and C. albicans ATCC90028), 1 strain for spontaneous mutation in SDD MIC level (C. albicans MYA4440), and 1 resistant strain (C. albicans ATCC96901). C. parapsilosis ATCC22019 and C. krusei ATCC6258 were used as quality control strains for susceptibility testing.

\section{Sensititre YeastOne Antifungal Susceptibility testing}

Twelve strains collected from Thammasat Hospital were subcultured on Sabouraud Dextrose Agar (SDA) and incubated at $35^{\circ} \mathrm{C}$ overnight. They were performed using Sensititre YeastOne (SYO) according to the manufacturer's instructions (Thermo Fisher Scientific, Cleveland, $\mathrm{OH}$, USA) for measuring MIC of 9 drugs included fluconazole, anidulafungin, amphotericin B, micafungin, caspofungin, 5-Flucytosine, posaconazole, voriconazole and itraconazole. The SYO plate was incubated at $35^{\circ} \mathrm{C}$ overnight and MIC endpoints were read after $24 \mathrm{~h}$ of incubation by the color changed from blue (negative, indicating no growth) to magenta (positive, indicating growth) [18].

\section{CLSI Broth microdilution method (CLSI BMD)}

Reference BMD testing was performed exactly as outlined in CLSI document M27-4 ${ }^{\text {th }}$ ed [19], with a final inoculum concentration of $0.5 \times 10^{3}$ to $2.5 \times 10^{3}$ cells $/ \mathrm{ml}$ and RPMI 1640 medium with $0.2 \%$ glucose, and incubation at $35^{\circ} \mathrm{C}$. MIC values were visually observed for the presence or absence of growth after $24 \mathrm{~h}$ (Candida and other yeast) and $72 \mathrm{~h}$ ( $C$. neoformans) of as the lowest concentration of drug that produced a prominent decrease in turbidity (ca. $50 \%$ growth reduction) relative to that of the drug-free control.

\section{Susceptibility breakpoints}


The MIC $(\mu \mathrm{g} / \mathrm{ml})$ interpretations follow the CLSI breakpoints for fluconazole $(\leq 2 \mu \mathrm{g} / \mathrm{ml}$; Susceptible, 4 $\mu \mathrm{g} / \mathrm{ml}$; Susceptible-dose dependent, $\geq 8 \mu \mathrm{g} / \mathrm{ml}$, Resistant). Interpretation of susceptibility was performed by applying the CBPs defined by CLSI [20]. In the CBPs absence, isolates were defined as having a wildtype or a non-wild-type drug susceptibility phenotype including amphotericin, 5-fucytosine, itraconazole and posaconazole according to the epidemiological cutoff values determined by SYO $[18,21]$.

\section{Selection of spontaneous mutants}

C. albicans MYA4440 isolates ( $\mathrm{MIC}=0.5 \mu \mathrm{g} / \mathrm{ml}$ ) and $\mathrm{G950}$ isolates $(\mathrm{MIC}=2 \mu \mathrm{g} / \mathrm{ml}$ ) were subcultured and incubated at $35^{\circ} \mathrm{C}$ overnight. The individual colony from each strain was used to start cultures in RPMI and grown overnight. One hundred microliters of overnight cultures $\left(5 \times 10^{8}\right.$ to $\left.1 \times 10^{9} \mathrm{CFU} / \mathrm{ml}\right)$ were streaked onto SDA plates with $(1,1.5,2$, and $4 \mu \mathrm{g} / \mathrm{ml})$ or without fluconazole and incubated at $35^{\circ} \mathrm{C}$ for 2 days. MIC was checked and resubcultured onto a fresh-passage until the MIC was $4 \mu \mathrm{g} / \mathrm{ml}$. The spontaneous mutation frequency rate was calculated as the ratio of viable colonies growing on drugcontaining plates over the starting inoculum. Mutant resistance phenotypes were confirmed by subculturing on SDA plates containing an amount of drug equivalent to that used for initial selection. Mutant strains were selected and evaluated by MIC and qRT-PCR.

\section{RNA extraction, cDNA synthesis and Quantitative real-time PCR (qRT-PCR)}

Each $C$. albicans isolate was suspended in $5 \mathrm{ml}$ of yeast nitrogen base (YNB) medium supplemented with $50 \mathrm{mM}$ glucose and incubated at $37^{\circ} \mathrm{C}$ in a shaker at $75 \mathrm{rpm}$ overnight to prepare each of the starters. After that, $500 \mu \mathrm{l}$ of the starter was transferred into a flask of $50 \mathrm{ml}$ containing fresh medium and incubated at $37^{\circ} \mathrm{C}$ in a shaker at $75 \mathrm{rpm}$ until $\mathrm{OD}_{600}=1.0$. Total RNA was extracted from the growth medium (at $0 \mathrm{~h}$ without fluconazole and after $24 \mathrm{~h}$ with $8 \mu \mathrm{g} / \mathrm{ml}$ of fluconazole addition) at midexponential (log) phase using the preparation of yeast RNA by extraction with hot acidic phenol. The concentration of RNA was measured using a NanoDrop 2000C spectrophotometer (Thermo Scientific). The RNA purity and integrity was evaluated by the ratio of absorbance 260 and $280 \mathrm{~nm}$. Run gel electrophoresis will be performed to verify that the RNA was intact. Total RNA was treated with TURBO DNA-free ${ }^{T M}$ Kit (Invitrogen) according to the manufacturer's instructions (RNA sample $>200 \mu$ g nucleic acid per $\mathrm{ml}$ ). cDNA was synthesized by reverse transcription from $1 \mu \mathrm{g}$ of total RNA using the iScript ${ }^{\mathrm{TM}}$ Reverse Transcription Supermix for RT-qPCR (Biorad) according to the manufacturer's instructions. The reaction protocols for cDNA synthesis were composed of priming step at $25^{\circ} \mathrm{C}$ for $5 \mathrm{~min}$, reverse transcription step at $46^{\circ} \mathrm{C}$ for $20 \mathrm{~min}$, and RT inactivation step at $95^{\circ} \mathrm{C}$ for $1 \mathrm{~min}$. The mRNA expression level was measured using quantitative real-time RT-PCR (qRT-PCR) following Watamoto's protocol [22]. The sequences primers (macrogen) of the genes CDR1, CDR2, MDR1, ERG11 [22], and PMA1 [23] in this study are listed in Table 3. qRT-PCR was performed in duplicate using iTaq ${ }^{\mathrm{TM}}$ Universal SYBR ${ }^{\circledR}$ Green Supermix (Biorad). Ten microlitres of PCR mix ( $5 \mu \mathrm{iTaq}^{\mathrm{TM}}$ Universal SYBR ${ }^{\circledR}$ Green Supermix, $1 \mu \mathrm{l}$ primer mix, $0.5 \mu \mathrm{l} \mathrm{cDNA}$ and $3.5 \mu \mathrm{l}$ DEPC water) was used for each gene and qRT-PCR was performed using the following cycling conditions: $95^{\circ} \mathrm{C}$ for $5 \mathrm{~min}$, followed by 40 cycles of $95^{\circ} \mathrm{C}$ for $15 \mathrm{~s}$ and $60^{\circ} \mathrm{C}$ for $30 \mathrm{~s}$. Fluorescence intensities were quantified using Bio-Rad ${ }^{\circledR}$ CFX96 ${ }^{\mathrm{TM}}$ (Biorad). The relative quantities of the 
target genes (CDR1, CDR2, MDR1 and ERG11) were normalized against PMA1 housekeeping gene expression (plasma membrane ATPase pump). The analyses of genes expressions were performed using the comparative $2^{(-\triangle \Delta C T)}$ method of relative quantification. The mRNA expression level of each target gene was analyzed. All results were presented by mean \pm SD. Statistical analyses were performed using GraphPad Prism 5.0 software. Results were compared using one way ANOVA followed by Bonferroni's post test; the results were considered statistically significant when the $p$ value was $<0.0001$.

\section{Detection of mass spectral analysis using MALDI-TOF MS}

Fresh-isolated colonies on SDA were cultured in RPMI 1640 medium, adjusted turbidity with 0.5 McFarland Standard, and incubated at $37^{\circ} \mathrm{C}$ overnight. Two milliliter $(\mathrm{ml})$ of suspension was added into RPMI 1640 medium with different fluconazole concentrations: 4 and $8 \mu \mathrm{g} / \mathrm{ml}$, and without fluconazole as a negative control. The suspension was incubated at $37^{\circ} \mathrm{C}$ and harvested at $6 \mathrm{~h}$ intervals and centrifuged and separated the pellets and the supernatant. The pellets were extracted with the modified of formic acid extraction following Bruker Daltonics recommended protocol for analysis of $C$. albicans protein spectrum. Briefly, $300 \mu \mathrm{l}$ of HPLC grade water and $700 \mu \mathrm{l}$ of ethanol were added, mixed with vortex for 1 min, and centrifuged 13,000 rpm for $3 \mathrm{~min}$. The solution was discarded after centrifugation. Then, the pellets were dried; $70 \%$ formic acid was added until covered the pellet, added the silica bead into the solution, and mixed with vortex for $2 \mathrm{~min}$. Acetonitrile was added the equal volume of $70 \%$ formic acid (ratio 1:1). The mixer was centrifuged at 13,000 rpm for $3 \mathrm{~min}$. One microliter of supernatant was spotted on the MALDI target plate and dried at room temperature (RT). One microliter of $2.5 \%$ TFA of a-Cyano-4hydroxycinnamic acid (HCCA) matrix was covered and dried at RT again. The sample was analyzed by MALDI-TOF MS (Bruker Daltonics, Bremen, Germany). The supernatant use for fluconazole spectrum analysis was dropped onto plate and dried, covered with $0.1 \%$ TFA of HCCA matrix, dried at RT, and analyzed by Bruker Autoflex Speed MALDI-TOF MS. The bacterial test standard (BTS), the ribosomal proteins of Escherichia coli strain DH5alpha, was used as a calibrator. The spectrum was measured by flexControl 3.3 software $[13,24]$ using linear mode $(2,000-20,000 \mathrm{~m} / \mathrm{z})$ for $C$. albicans spectrum and using reflector mode $(0-3,500 \mathrm{~m} / \mathrm{z})$ for fluconazole spectrum. Finally, the data was processed and compared using flexAnalysis.

\section{Results}

\section{MIC level of control strains, clinical strains and spontaneous mutant strains}

Of the FLC susceptibility testing with 4 reference strains, C. albicans MYA2876, C. albicans ATCC90028 and $C$. albicans MYA4440 were susceptible (S) to FLC at MIC level of $0.25,0.25$ and $0.5 \mu \mathrm{g} / \mathrm{ml}$, respectively, whereas $C$. albicans ATCC96901 was resistant to FLC at MIC level of $\geq 64 \mu \mathrm{g} / \mathrm{ml}$. With spontaneous mutation process up to 10 consecutive serial passages, $C$. albicans MYA4440 mutant ( $\triangle$ MYA4440) were susceptible-dose dependent to FLC $(\mathrm{MIC}=4 \mu \mathrm{g} / \mathrm{ml})$, while $\mathrm{G} 950$ strain was not change in MIC level. All 27 clinical strains of $C$. albicans were susceptible to FLC at MIC range $0.5-2 \mu \mathrm{g} / \mathrm{ml}$ (Table 1). Twelve strains collected from Thammasat University Hospital using Sensititre YeastOne 
(SYO) were susceptible to fluconazole, voriconazole, anidulafungin, caspofungin and micafungin when applying CLSI CBPs. When applying the epidemiological cutoff values (ECVs), all the isolates had wild type phenotype drug susceptibility to amphotericin, 5-flucytosine, posaconazole and itraconazole (Table 2).

\section{CDR efflux pump overexpression}

Figure $1 \mathrm{a}$ and $1 \mathrm{~b}$ indicated $C D R$ efflux pump overexpression in $C$. albicans strains, the SDD ( $\triangle \mathrm{MYA4440)}$ and R (ATCC96901) strains significantly overexpressed both CDR1and CDR2 ( $\mathrm{p}$-value $<0.0001$ ) at higher level than S strains. Compared to $C$. albicans MYA4440 (wild type) strain (MIC $0.5 \mu \mathrm{g} / \mathrm{ml}$ ), its mutant strain ( $\triangle \mathrm{MYA4440)}$ ) at MIC level of $4 \mu \mathrm{g} / \mathrm{ml}$ expressed $C D R 1$ and $C D R 2$ at higher level. After incubation with $8 \mu \mathrm{g} / \mathrm{ml}$ of FLC for $24 \mathrm{~h}$, the SDD strain ( $\triangle$ MYA4440) and R strain (ATCC96901) significantly overexpressed CDR1 (p-value $<0.0001$ ) but significantly repressed CDR2 ( $\mathrm{p}$-value $<0.0001$ ), while $\mathrm{S}$ strains (MYA2876, ATCC90028 and MYA4440) and all clinical S strains were nonsignificantly expressed CDR1 and CDR2 (p-value >0.0001).

\section{MDR1 efflux pump overexpression}

MDR1 expression were not significantly different among S (MYA2876), SDD ( $\triangle$ MYA4440) and R (ATCC96901) strains ( -value > 0.0001) (Fig. 1c). Without the addition of FLC, some SDD, R and some of $S$ strains including $3 \mathrm{~S}$ reference strain and some $\mathrm{S}$ clinical strains expressed MDR1. After incubation with $8 \mu \mathrm{g} / \mathrm{ml}$ of FLC for $24 \mathrm{~h}$, almost strains were not significantly changed in MDR1 expression. Some strains (Y59, S13, G189, H282, G627, G1002, R237, S261 and G582) were observed MDR1 overexpression. Nonetheless, except 3 strains (Y4, $\mathrm{Y} 5$ and $\mathrm{Y} 63)$ showed significant result as MDR1 repression ( $p$-value $<0.0001$ ).

\section{ERG11 target enzyme overexpression}

Figure 1d explained ERG11 overexpression. No statistically significant differences of ERG11 expression ( $p$-value > 0.0001) were seen among all strains. After incubation with $8 \mu \mathrm{g} / \mathrm{ml}$ of FLC for $24 \mathrm{~h}$, all strains showed increased ERG11 expression. The 15 strains of clinical susceptible strains (55.56\%) were significantly increased ERG11 expression (p-value $<0.0001$ ), while 12 strains $(44.44 \%)$ and all control strains were not significantly different ( $p$-value $>0.0001$ ).

\section{The protein spectrum related to the fluconazole resistance of $C$. albicans in control standard strains}

This experiment was to observe FLC mass spectral change among S, SDD and R control strains after incubation with 4 and $8 \mu \mathrm{g} / \mathrm{ml}$ for $6 \mathrm{~h}$. Before initiating the experiment, the quality control of reagent, microorganisms, drug, and incubation time period was investigated as shown in Fig. 2 and Fig. 3. Figure 2 showed the mass spectral range $0-600 \mathrm{~m} / \mathrm{z}$ of $0.1 \%$ HCCA, FLC S C. albicans strain, FLC SDD C. albicans strain, FLC R C. albicans strain and FLC. The mass spectral intensity of FLC was appeared the spectrum position at $307 \mathrm{~m} / \mathrm{z}$, while those of $0.1 \%$ HCCA and FLC S, SDD and R strains was not seen any peak like FLC mass spectrum. After testing FLC incubation for 2, 4 and $6 \mathrm{~h}$, the mass spectral intensity of FLC was 
present all the time of study period (Fig. 3). This advocated that the incubation of studied time period does not affect the FLC mass spectrum. After testing with $S$ and $R C$. albicans strains by incubation with 4 and $8 \mu \mathrm{g} / \mathrm{ml}$ for $6 \mathrm{~h}$, FLC mass spectral intensity of $8 \mu \mathrm{g} / \mathrm{ml} \mathrm{FLC} \mathrm{addition} \mathrm{was} \mathrm{higher} \mathrm{than} \mathrm{that} \mathrm{of} 4$ $\mu \mathrm{g} / \mathrm{ml} \mathrm{FLC} \mathrm{addition.} \mathrm{No} \mathrm{FLC} \mathrm{hydrolysis} \mathrm{was} \mathrm{detected} \mathrm{in} \mathrm{this} \mathrm{experiment} \mathrm{(Fig.} \mathrm{4).} \mathrm{Spectrum} \mathrm{position} \mathrm{at} 307$ $\mathrm{m} / \mathrm{z}$ of fluconazole can found in every fluconazole addition condition which is at $8 \mu \mathrm{g} / \mathrm{ml}$ of fluconazole concentration, the intensity will higher than at $4 \mu \mathrm{g} / \mathrm{ml}$ of fluconazole concentration (Fig. 4). This suggested that FLC hydrolysis is not the mechanism of action of FLC in C. albicans. Therefore, we cannot use FLC mass spectrum position to determine among FLC S, SDD and R strains.

Then, the spectrum pattern of $C$. albicans at mass spectral range 2,000-20,000 m/z was investigated after incubation with 0 (control), 4 and $8 \mu \mathrm{g} / \mathrm{ml}$ for $6 \mathrm{~h}$ (Fig. 5). Interestingly, the change of mass spectral intensity at range $3376-3382 \mathrm{~m} / \mathrm{z}$ (major peak) was significantly related to the FLC susceptibility to $\mathrm{S}$, SDD and R reference strains. Compared to $0 \mu \mathrm{g} / \mathrm{ml}$ of FLC, 3 types of major peak were: 1 ) increased 4 and $8 \mu \mathrm{g} / \mathrm{ml}$ (S strain), 2) decreased at $4 \mu \mathrm{g} / \mathrm{ml}$ and increase at $8 \mu \mathrm{g} / \mathrm{ml}$ (SDD strain), and 3) slightly decreased or no change at 4 and $8 \mu \mathrm{g} / \mathrm{ml}$ (R strain). After observing the major peak of all 27 clinical strains, their results showed $S$ pattern like $S$ reference strain (data not shown). This is in line with FLC MIC recognized as susceptible (MIC range $0.25-2 \mu \mathrm{g} / \mathrm{ml}$ ) and no change in CDR1 and CDR2 overexpression.

\section{Discussion}

Here, all $C$. albicans strains compared to MIC level in this study, CDR1 and CDR2 genes expression are only related to FLC SDD ( $\triangle$ MYA4440) and R strains (ATCC96901) without FLC addition. These results disclosure the resistance mechanism via increased efflux of the drug from cells by $A B C$ transporters. The $\mathrm{R}$ strain has a higher expression of $C D R 1$ and $C D R 2$ than SDD strain. Although only 2 samples including 1 spontaneous mutant SDD strain and $1 \mathrm{R}$ reference strain are available in this study, the results are in line with previous reported that high expression levels of $C D R$ efflux genes is a major mechanism for fluconazole resistance in C. albicans [6]. MDR1 expression in our study was not significantly different among S, SDD and R strains ( $p$-value >0.0001). This may support the previous study revealed that in planktonic cell $C D R$ displays higher expression level after $24 \mathrm{~h}$ incubation, while MDR1 expression was even higher after $48 \mathrm{~h}[10]$. Contradictory to our study revealed that $C D R 1$ overexpressed in SDD and $\mathrm{R}$ strains after $24 \mathrm{~h}$ incubation with FLC, previous study observed that FLC could induce the expression of $C D R 1, C D R 2$ and MDR1 [10]. For ERG11 expression, all strains showed increased after incubation with 8 $\mu \mathrm{g} / \mathrm{ml} \mathrm{FLC} \mathrm{for} 24 \mathrm{~h}$. This supported the previous report that the expression of $E R G 11$ were increased after antifungal treatment in susceptible and non-susceptible to azole isolates [11]. Considering to determine FLC susceptibility to $C$. albicans with MALDI-TOF MS, we found only one spectrum at position 3376$3382 \mathrm{~m} / \mathrm{z}$ that was linked with the FLC resistance of $C$. albicans after incubation with 4 and $8 \mu \mathrm{g} / \mathrm{ml} \mathrm{FLC}$ for $6 \mathrm{~h}$. Indeed, by allowing a peak position's tolerance of $\pm 3 \mathrm{~m} / \mathrm{z}$ were overcome the small spectral variations and able to assess as $C$. albicans housekeeping peaks [12]. The drug concentration and incubation time period seem be a major factor for each Candida species to observe a major peak for differentiating $\mathrm{S}$ strain from R strain. In view of the study of Paul, both the resistant and susceptible $C$. tropicalis isolates showed spectral changes after $4 \mathrm{~h}$ when challenged with $128 \mu \mathrm{g} / \mathrm{ml}$ and $1 \mu \mathrm{g} / \mathrm{ml} \mathrm{FLC}$, 
respectively [13]. In our study, the major mass spectral intensity change was clearly seen after $6 \mathrm{~h}$ incubation with FLC. As reported by Vatanshenassan using MBT ASTRA prototype software, the discrimination between resistant and susceptible strains was accurately detected in $6 \mathrm{~h}$ [14]. Furthermore, the previous report explained that prolonged exposures antifungal drug in some isolates were allowed to increasing correctly identifying between S and R strains [8]. Together with the condition of drug concentration and incubation time period, the method processed in each study could support for determining FLC resistant $C$. albicans strains and also finding different major mass spectrum. For example, the study of Marinach [15], MPCC determination was used to decide FLC $C$. albicans resistance strains with MALDI-TOF MS. Their study was concluded that the most suitable of FLC concentrations were 2 and $4 \mu \mathrm{g} / \mathrm{ml}$ and significant spectral intensity range 5,800-7,600 m/z were used to detect FLC resistance. Six spectrums in this range could detect the different of FLC resistance. Many studies used the difference of the operating procedure including MPCC method $[13,15]$, composite correlation index (CCI) values approach $[8,16,17]$, matrix-assisted laser desorption ionization Biotyper antibiotic susceptibility test rapid assay (MBT-ASTRA) $[1,9]$ and machine-learning algorithms with the most robust pipeline of analysis [12]. Now, we disclosure a specific mass spectrum for analyzing FLC S, SDD and R strain for $C$. albicans with their resistance related to $C D R$ expression efflux pump. For approving our results followed by the same protocol, more 27 C. albicans strains from clinical samples at various sites of 27 patients were investigated the major peak type by MALDI-TOF MS and FLC MIC by BMD. All strains presented a novel mass spectral intensity at range 3376-3382 m/z (major peak) and the major peak type was observed as $S$ type and interpreted MIC level as susceptible (data not shown). Unfortunately, we could not find FLC SDD or R $C$. albicans strain from blood cultures of candidemic patients in the University Hospitals during our study. Moreover, we followed the protocol for determining in $48 C$. tropicalis strains including FLC S, SDD and R strains from blood cultures of 48 patients. Unsuccessfully, the major peak type could not use for differentiating $C$. tropicalis (data not shown). With this reason, this major peak type is specific for determining FLC susceptibility testing to $C$. albicans.

\section{Conclusions}

Overall, we discover a novel mass spectral intensity at range $3376-3382 \mathrm{~m} / \mathrm{z}$ (major peak) related to the FLC susceptibility to S, SDD and R strains specific for $C$. albicans. The FLC resistance mechanisms of $C$. albicans associated with CDR1 and CDR2 expression (via increased efflux of the drug from cells by $A B C$ transporters) may possibly effect the change of mass spectral intensity at range $3376-3382 \mathrm{~m} / \mathrm{z}$. The results demonstrate that the MALDI-TOF MS may be used to simultaneously classify Candida species and predict FLC resistant $C$. albicans strains associated with $C D R 1$ and $C D R 2$ overexpression. However, the limitations of our study including the small number of clinical $C$. albicans strains, the shortage of SDD and R clinical $C$. albicans strains, and unproven the protein spectrum at position 3376-3382 $\mathrm{m} / \mathrm{z}$. Therefore, further studies are essential to clarify the methodology and improve the reliability of this assay for routine diagnosis.

\section{Abbreviations}


C. albicans: Candida albicans, MALDI-TOF MS: Matrix-assisted laser desorption ionization-time of flight mass spectrometry; Real-time PCR: Real-time polymerase chain reaction; CDR: Candida drug resistance; $M D R$ : multidrug resistance; ERG: ergosterol biosynthesis; FLC: Fluconazole; S: Susceptible; R: Resistant; SDD: Susceptible-dose dependent; mRNA: messenger ribonucleic acid; MIC: minimal inhibitory concentration; ABC transporters: ATP-binding cassette transporters; MFS: Major facilitator superfamily; BMD: broth microdilution; AFST: antifungal susceptibility test; CLSI: Clinical and Laboratory Standards Institute; EUCAST: European Committee on Antibiotic Susceptibility Testing; SYO: Sensititre YeastOne; CBPs: clinical breakpoints; ECVs: epidemiological cutoff values; MPCC: minimal profile changing concentration; CCl: Composite correlation index; MBT-ASTRA: matrix-assisted laser desorption ionization Biotyper antibiotic susceptibility test rapid assay; SDA: Sabouraud Dextrose Agar; RPMI 1640 medium: Roswell Park Memorial Institute 1640 culture medium; cDNA: complementary deoxyribonucleic acid; qRT-PCR: quantitative real-time polymerase chain reaction; YNB: yeast nitrogen base; OD: optical density; RT-qPCR: quantitative reverse transcription polymerase chain reaction; RT inactivation: reverse transcriptase inactivation; PMA7: plasma membrane ATPase pump; DEPC: Diethyl pyrocarbonate; HPLC: high performance liquid chromatography; RT: room temperature; TFA: Trifluoroacetic acid; HCCA: aCyano-4-hydroxycinnamic acid; BTS: bacterial test standard

\section{Declarations}

\section{Ethics approval and consent to participate}

The research was approved by Human Ethical Committee of Thammasat University (054/2561) and Navamindradhiraj University (04/2018) and also accredited by Biosafety Committee of Thammasat University (038/2561).

\section{Consent for publication}

Not applicable.

\section{Availability of data and material}

All the data required is included in the manuscript.

\section{Competing interests}

The authors declare that they have no competing interests.

\section{Funding}

This work was supported by grants from the National Research Council of Thailand, 2019. The authors gratefully acknowledge the financial support provided by Thammasat University Research Fund under the TU Research Scholar 2018, Contract No. 67/2561.

\section{Authors' contributions}


Chanika Maenchantrarath carried out the study design, sample preparation, experiments, and data analysis and wrote the initial draft of the manuscript. Pradchama Khumdee helps some parts of MALDITOF MS experiment. Seksun Samosornsuk gave guidance and support. Narissara Mungkornkaew identified and collected some yeast samples. Worada Samosornsuk gave advices, contributed to the analysis of the evaluation of the study, and was a major contributor in writing the manuscript. All authors read and approved the final manuscript.

\section{Acknowledgements}

The authors gratefully acknowledge the National Research Council of Thailand, 2019, and Thammasat University Research Fund under the TU Research Scholar 2018, Contract No. 67/2561, for financial support.

\section{References}

1. Delavy M, Dos Santos AR, Heiman CM, Coste AT. Investigating Antifungal Susceptibility in Candida Species With MALDI-TOF MS-Based Assays. Front Cell Infect

Microbiol. 2019;9:19. http://doi.org/10.3389/fcimb.2019.00019.

2. Guinea J. Global trends in the distribution of Candida species causing candidemia. Clin Microbiol Infect. 2014;20 Suppl 6:5-10. http://doi.org/10.1111/1469-0691.12539.

3. Cowen LE, Nantel A, Whiteway MS, Thomas DY, Tessier DC, Kohn LM, et al. Population genomics of drug resistance in Candida albicans. Proc Natl Acad Sci U S A. 2002;99(14):9284-

9. http://doi.org/10.1073/pnas.102291099.

4. Rosana Y, Yasmon A, Lestari DC. Overexpression and mutation as a genetic mechanism of fluconazole resistance in Candida albicans isolated from human immunodeficiency virus patients in Indonesia. $\mathrm{J}$ Med Microbiol. 2015;64(9):1046-52. http://doi.org/10.1099/jmm.0.000123.

5. Berkow EL, Lockhart SR. Fluconazole resistance in Candida species: a current perspective. Infect Drug Resist. 2017;10:237-45. http://doi.org/10.2147/IDR.S118892.

6. Khosravi Rad K, Falahati M, Roudbary M, Farahyar S, Nami S. Overexpression of MDR-1 and CDR2 genes in fluconazole resistance of Candida albicans isolated from patients with vulvovaginal candidiasis. Curr Med Mycol. 2016;2(4):24-9. http://doi.org/10.18869/acadpub.cmm.2.4.24.

7. De Carolis E, Paoletti S, Nagel D, Vella A, Mello E, Palucci I, et al. A rapid diagnostic workflow for cefotaxime-resistant Escherichia coli and Klebsiella pneumoniae detection from blood cultures by MALDITOF mass spectrometry. PloS One. 2017;12(10):e0185935. http://doi.org/10.1371/journal.pone.0185935.

8. Vella A, De Carolis E, Mello E, Perlin DS, Sanglard D, Sanguinetti M, et al. Potential Use of MALDI-ToF Mass Spectrometry for Rapid Detection of Antifungal Resistance in the Human Pathogen Candida 
glabrata. Sci Rep. 2017;7(1):9099. http://doi.org/10.1038/s41598-017-09329-4.

9. Vatanshenassan M, Boekhout T, Lass-Flörl C, Lackner M, Schubert S, Kostrzewa M, et al. Proof of Concept for MBT ASTRA, a Rapid Matrix-Assisted Laser Desorption lonization-Time of Flight Mass Spectrometry (MALDI-TOF MS)-Based Method To Detect Caspofungin Resistance in Candida albicans and Candida glabrata. J Clin Microbiol. 2018;56(9):00420-18. http://doi.org/10.1128/JCM.00420-18.

10. Shi C, Liu J, Li W, Zhao Y, Meng L, Xiang M. Expression of fluconazole resistance-associated genes in biofilm from 23 clinical isolates of Candida albicans. Braz J Microbiol. 2019;50(1):157-

63. http://doi.org/10.1007/s42770-018-0009-2.

11. Silva MC, Cardozo Bonfim Carbone D, Diniz PF, Freitas Fernandes F, Fuzo CA, Santos Pereira Cardoso Trindade C, et al. Modulation of ERG Genes Expression in Clinical Isolates of Candida tropicalis Susceptible and Resistant to Fluconazole and Itraconazole. Mycopathologia. 2020;185(4):675684. http://doi.org/10.1007/s11046-020-00465-6.

12. Delavy M, Cerutti L, Croxatto A, Prod'hom G, Sanglard D, Greub G, et al. Machine Learning Approach for Candida albicans Fluconazole Resistance Detection Using Matrix-Assisted Laser Desorption/lonization Time-of-Flight Mass Spectrometry. Front Microbiol. 2020;10:3000. http://doi.org/10.3389/fmicb.2019.03000.

13. Paul S, Singh P, A S S, Rudramurthy SM, Chakrabarti A, Ghosh AK. Rapid detection of fluconazole resistance in Candida tropicalis by MALDI-TOF MS. Med Mycol. 2017;56(2):234-

41. http://doi.org/10.1093/mmy/myx042.

14. Florio W, Baldeschi L, Rizzato C, Tavanti A, Ghelardi E, Lupetti A. Detection of Antibiotic-Resistance by MALDI-TOF Mass Spectrometry: An Expanding Area. Front Cell Infect Microbiol. 2020;10:572909. http://doi.org/10.3389/fcimb.2020.572909.

15. Marinach C, Alanio A, Palous M, Kwasek S, Fekkar A, Brossas JY, et al. MALDI-TOF MS-based drug susceptibility testing of pathogens: the example of Candida albicans and fluconazole. Proteomics. 2009;9(20):4627-31. http://doi.org/10.1002/pmic.200900152.

16. Vella A, De Carolis E, Vaccaro L, Posteraro P, Perlin DS, Kostrzewa M, et al. Rapid antifungal susceptibility testing by matrix-assisted laser desorption ionization-time of flight mass spectrometry analysis. J Clin Microbiol. 2013;51(9):2964-9. http://doi.org/10.1128/JCM.00903-13.

17. De Carolis E, Vella A, Florio AR, Posteraro P, Perlin DS, Sanguinetti M, et al. Use of matrix-assisted laser desorption ionization-time of flight mass spectrometry for caspofungin susceptibility testing of Candida and Aspergillus species. J Clin Microbiol. 2012;50(7):2479-83. http://doi.org/10.1128/JCM.00224-12.

18. Espinel-Ingroff A, Turnidge J, Alastruey-Izquierdo A, Botterel F, Canton E, Castro C, et al. MethodDependent Epidemiological Cutoff Values for Detection of Triazole Resistance in Candida and Aspergillus 
Species for the Sensititre YeastOne Colorimetric Broth and Etest Agar Diffusion Methods. Antimicrob Agents Chemother. 2019;63(1):e01651-18. http://doi.org/10.1128/AAC.01651-18.

19. Wayne PA. Reference method for broth dilution antifungal susceptibility testing of yeasts. $4^{\text {th }}$ edition, CLSI document M27-4 ${ }^{\text {th }}$ ed. Clinical and Laboratory Standards Institute. 2017.

20. Berkow EL, Lockhart SR, Ostrosky-Zeichner L. Antifungal Susceptibility Testing: Current Approaches. Clin Microbiol Rev. 2020;33(3):e00069-19. http://doi.org/10.1128/CMR.00069-19.

21. Cantón E, Pemán J, Hervás D, Iñiguez C, Navarro D, Echeverría J, et al. Comparison of three statistical methods for establishing tentative wild-type population and epidemiological cutoff values for echinocandins, amphotericin B, flucytosine, and six Candida species as determined by the colorimetric Sensititre YeastOne method. J Clin Microbiol. 2012;50(12):3921-6. http://doi.org/10.1128/JCM.0173012.

22. Watamoto T, Samaranayake LP, Egusa H, Yatani H, Seneviratne CJ. Transcriptional regulation of drugresistance genes in Candida albicans biofilms in response to antifungals. J Med Microbiol. 2011;60:12417. http://doi.org/10.1099/jmm.0.030692-0.

23. Nailis H, Coenye T, Van Nieuwerburgh F, Deforce D, Nelis HJ. Development and evaluation of different normalization strategies for gene expression studies in Candida albicans biofilms by real-time PCR. BMC Mol Biol. 2006;7:25. http://doi.org/10.1186/1471-2199-7-25.

24. Pfaller MA, Chaturvedi V, Diekema DJ, Ghannoum MA, Holliday NM, Killian SB, et al. Clinical Evaluation of the Sensititre YeastOne Colorimetric Antifungal Panel for Antifungal Susceptibility Testing of the Echinocandins Anidulafungin, Caspofungin, and Micafungin. J Clin Microbiol. 2008;46(7):2155-9. http://doi.org/10.1128/JCM.00493-08.

\section{Tables}

Due to technical limitations, table 1-3 is only available as a download in the Supplemental Files section.

\section{Figures}


a) $C D R 1$

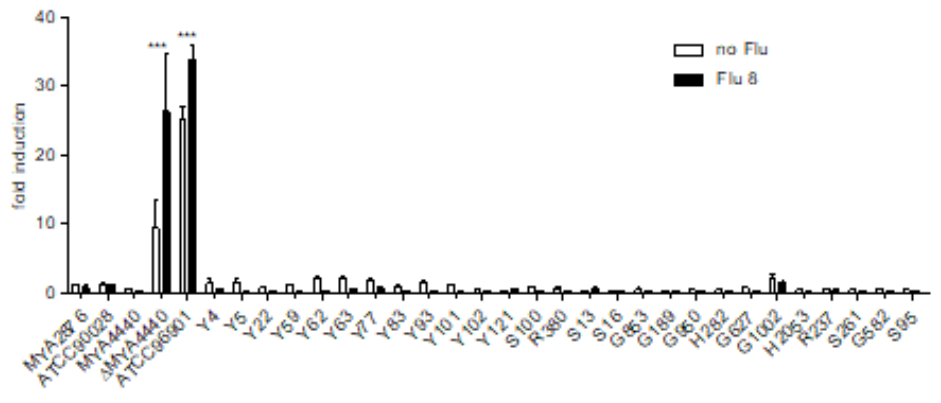

b) $C D R 2$

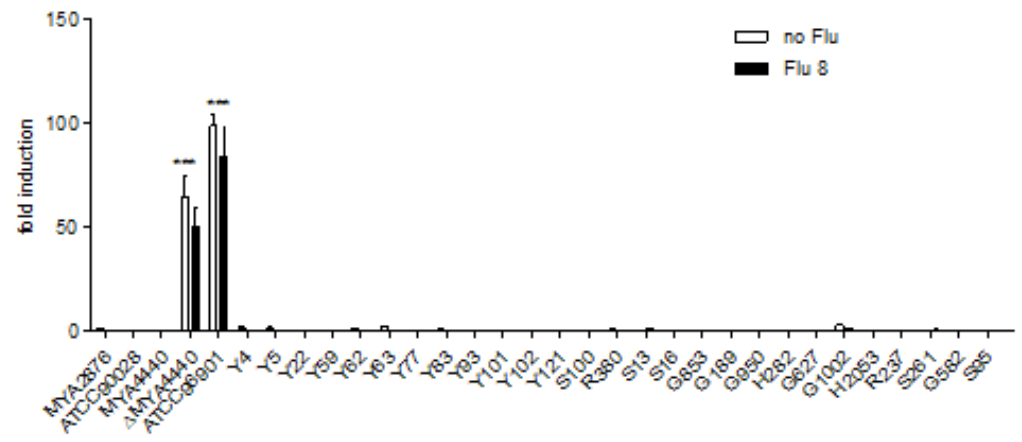

c) MDR1

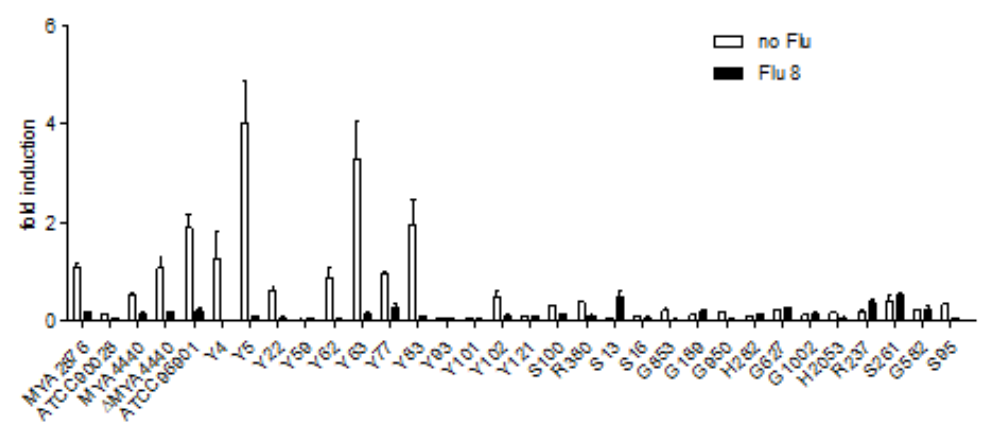

d) ERG11

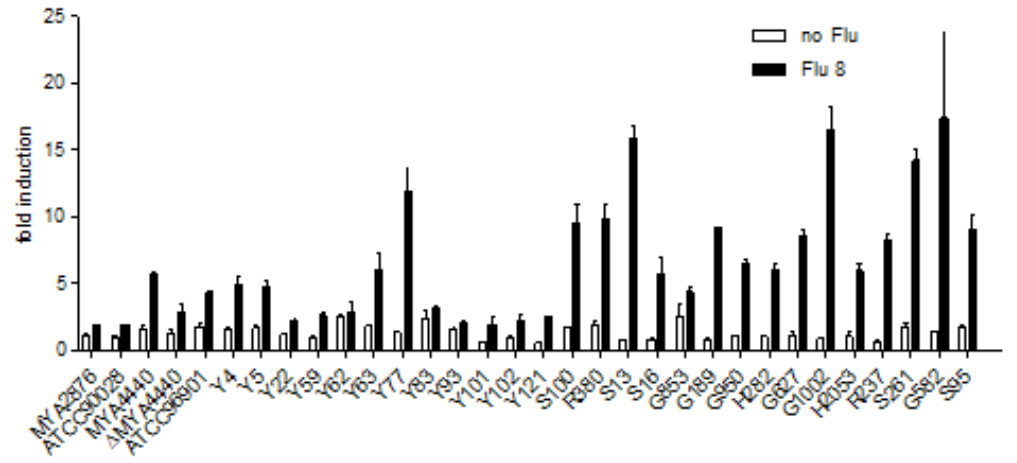

Figure 1

The mRNA expression of target genes a) CDR1 b) CDR2 c) MDR1 and d) ERG11 in C. albicans susceptible strain ( $n=30$; MYA2876, ATCC90028, MYA4440, and 27 clinical strains), fluconazole susceptible-dose dependent strain $(n=1 ; \triangle M Y A 4440)$ and resistant strain $(n=1 ;$ ATCC96901), comparison between without fluconazole condition and after fluconazole $8 \mu \mathrm{g} / \mathrm{ml}$ addition for 24 hours 


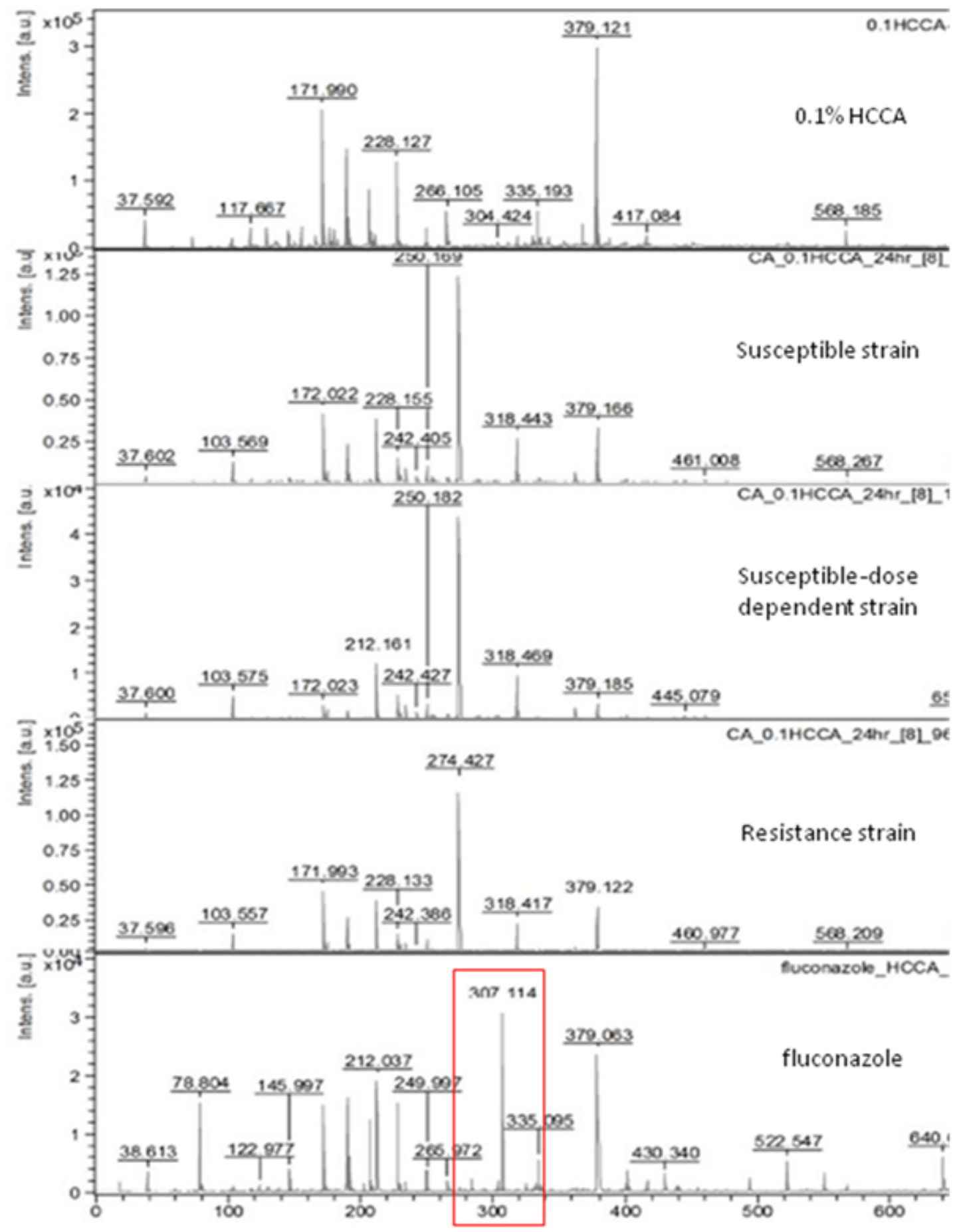

\section{Figure 2}

The spectrum of control $0.1 \%$ HCCA, fluconazole susceptible, susceptible-dose dependent strain, resistant C. albicans strains, and fluconazole position at $307 \mathrm{~m} / \mathrm{z}$ (red box) (from top to bottom) using the reflector mode 


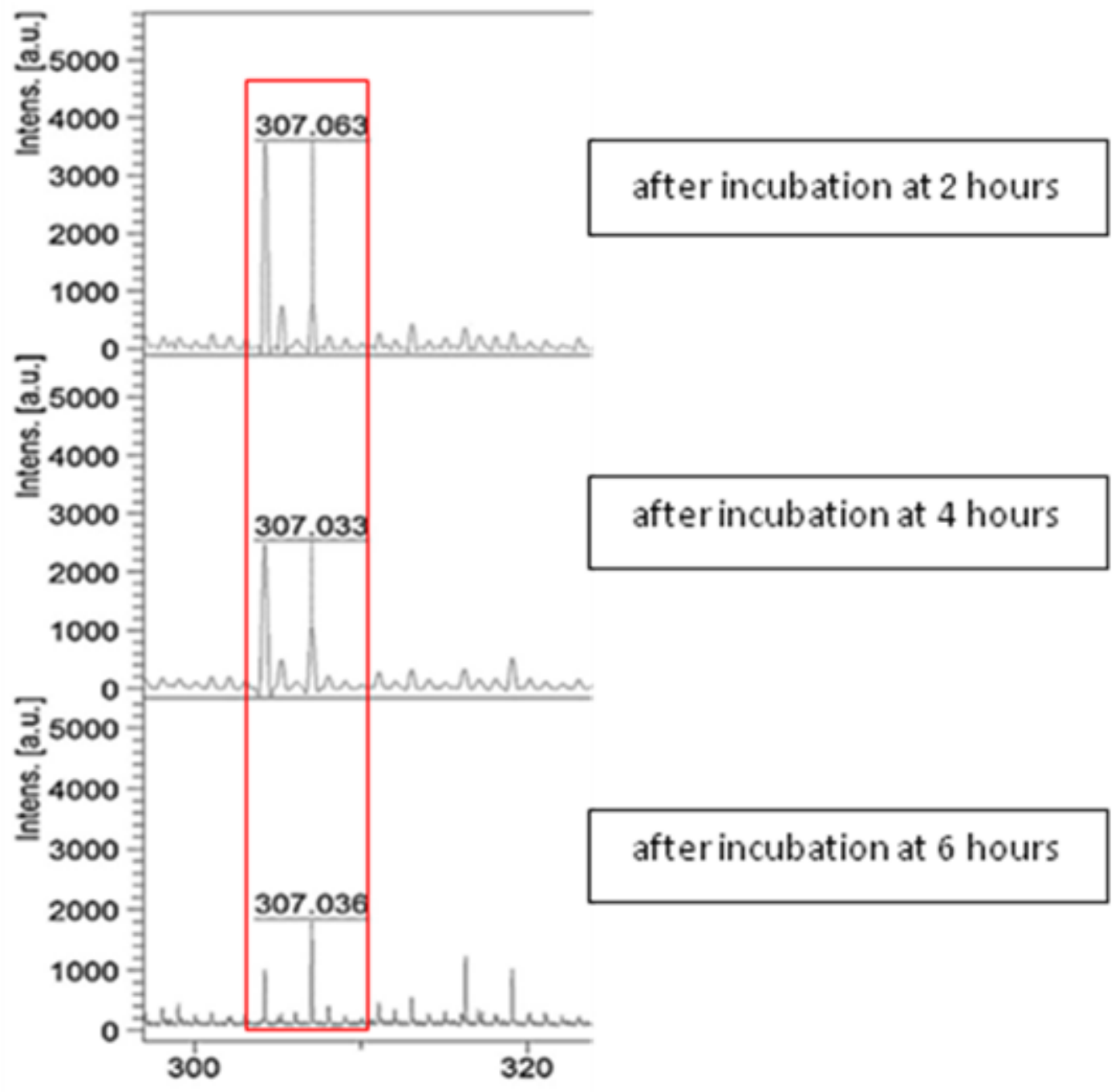

Figure 3

The spectrum of fluconazole position at $307 \mathrm{~m} / \mathrm{z}$ (red box) after incubation at 2, 4 and 6 hours (from top to bottom) using the reflector mode 


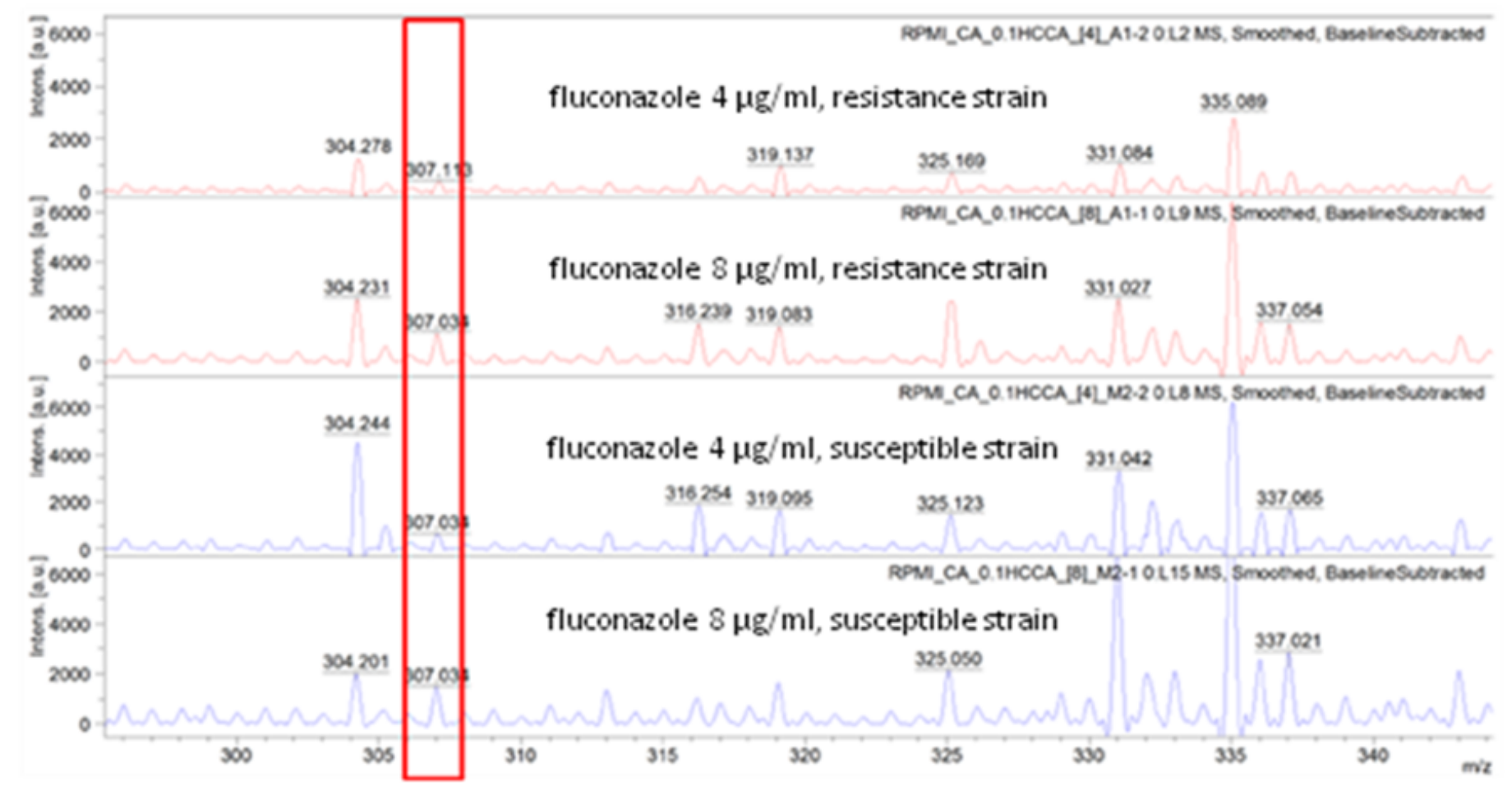

\section{Figure 4}

The comparison of fluconazole spectrum, position at $307 \mathrm{~m} / \mathrm{z}$ (red box), after incubation at fluconazole concentration 4 and $8 \mu \mathrm{g} / \mathrm{ml}$ of resistance strain and susceptible strain, respectively (from top to bottom)

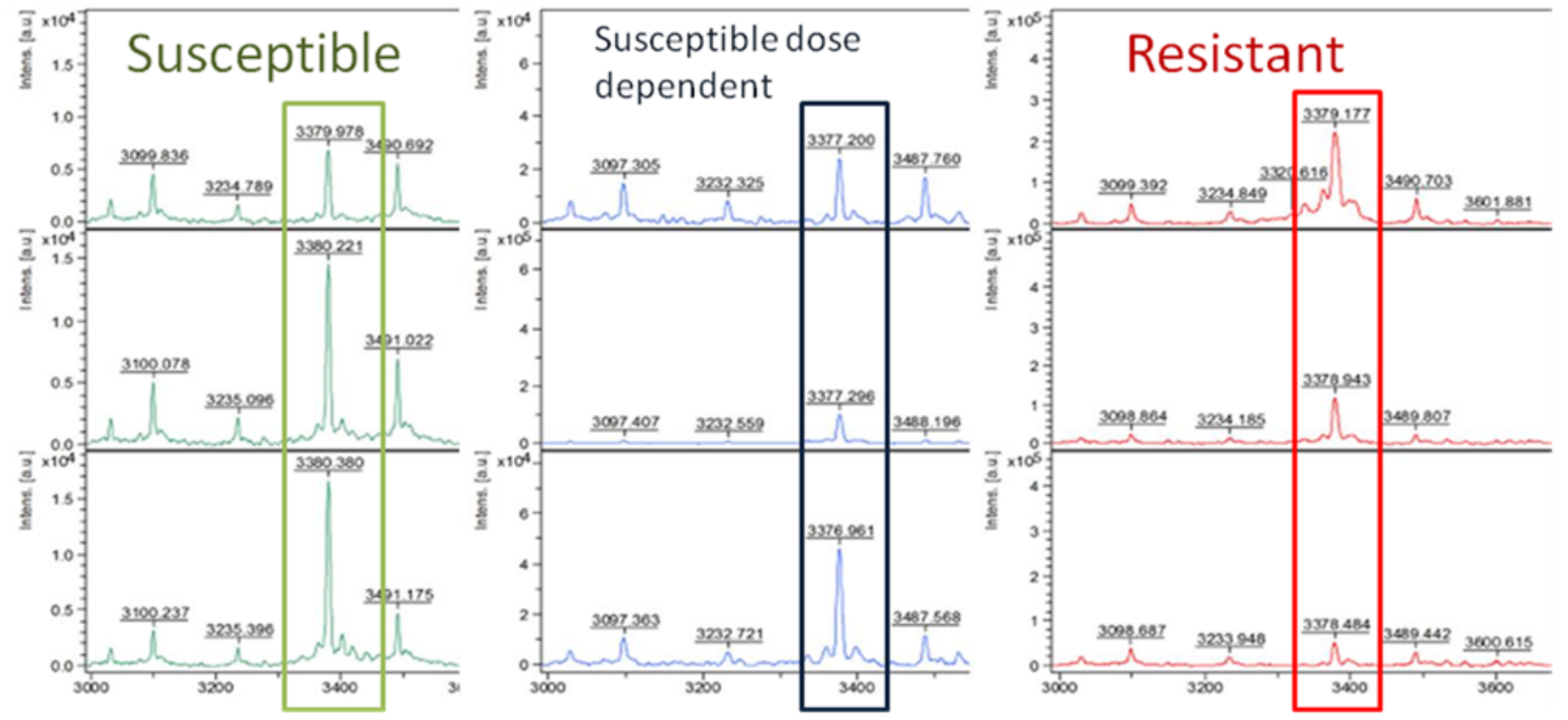

Figure 5 
The spectrum intensity change at position $3376-3382 \mathrm{~m} / \mathrm{z}$ of fluconazole-susceptible (green box) fluconazole-susceptible-dose dependent (blue box) and fluconazole-resistant (red box) C. albicans strains.

\section{Supplementary Files}

This is a list of supplementary files associated with this preprint. Click to download.

- MaenchantrarathTable1.pdf

- MaenchantrarathTable2.pdf

- MaenchantrarathTable3.pdf 